\title{
Linear algebra of the Lucas matrix
}

\author{
Nurettin $\operatorname{Irmak}^{1}$ (D), Cahit Köme*2 (1) \\ ${ }^{1}$ Konya Technical University, Faculty of Engineering and Natural Science, Department of Fundamental \\ Science, Konya, Turkey \\ ${ }^{2}$ Nevşehir Hacı Bektaş Veli University, Department of Information Technology, Turkey
}

\begin{abstract}
In this paper, we give the factorizations of the Lucas and inverse Lucas matrices. We also investigate the Cholesky factorization of the symmetric Lucas matrix. Moreover, we obtain the upper and lower bounds for the eigenvalues of the symmetric Lucas matrix by using some majorization techniques.
\end{abstract}

Mathematics Subject Classification (2020). 15A23, 11B39, 15A18, 15A42

Keywords. Lucas matrix, Cholesky factorization, eigenvalues, majorization

\section{Introduction}

The Fibonacci and Lucas numbers play an important role in various areas such as mathematics, physics, computer science and related fields. For $n \geqslant 0$, the Fibonacci and Lucas numbers are defined by following recurrence relations

$$
F_{n+2}=F_{n+1}+F_{n}, \quad F_{0}=0, \quad F_{1}=1
$$

and

$$
L_{n+2}=L_{n+1}+L_{n}, \quad L_{0}=2, \quad L_{1}=1,
$$

respectively. For more information about these numbers and their properties, we refer to the book [6].

Matrix factorizations provide considerable convenience while performing some difficult computations. Recently, several researchers have studied the factorizations of some matrices whose elements are the Fibonacci numbers. For example, Lee et al. investigated the factorizations and eigenvalues of the Fibonacci and symmetric Fibonacci matrices which are defined by

$$
\mathscr{F}_{n}=\left[f_{i j}\right]= \begin{cases}F_{i-j+1}, & i-j+1 \geqslant 0 \\ 0, & i-j+1<0\end{cases}
$$

and

$$
\mathscr{Q}_{n}=\left[q_{i j}\right]=\left[q_{j i}\right]=\left\{\begin{array}{ll}
\sum_{k=1}^{i} F_{k}^{2}, & i=j \\
q_{i, j-2}+q_{i, j-1}, & i+1 \leqslant j
\end{array},\right.
$$

respectively [7]. Kılıç and Taşçı, presented factorizations and eigenvalues of the Pell matrix and symmetric Pell matrix whose elements are the Pell numbers [5]. They also

\footnotetext{
*Corresponding Author.

Email addresses: nirmak@ohu.edu.tr (N. Irmak), cahit@nevsehir.edu.tr (C. Köme)

Received: 31.05.2020; Accepted: 15.09.2020
} 
give some bounds for the eigenvalues of the symmetric Pell matrix. Motivated by the above works, several authors studied factorization of the lower triangular matrices and investigated some special type matrices (see $[1,2,5,8,10]$ ).

Besides these works, in this paper, we define the Lucas matrix and the symmetric Lucas matrix as follows:

$$
\mathscr{L}_{n}=\left[l_{i j}\right]=\left\{\begin{array}{cc}
L_{i-j+1}, & i-j+1 \geqslant 0 \\
0, & i-j+1<0
\end{array},\right.
$$

and

$$
\mathscr{R}_{n}=\left[r_{i j}\right]=\left[r_{j i}\right]=\left\{\begin{array}{cc}
\sum_{k=1}^{i} L_{k}^{2}, & i=j \\
r_{i, j-2}+r_{i, j-1}+2, & i+1=j \\
r_{i, j-2}+r_{i, j-1}, & i+2 \leqslant j
\end{array},\right.
$$

where $r_{1,0}=0$. For example,

$$
\mathscr{L}_{5}=\left[\begin{array}{ccccc}
1 & 0 & 0 & 0 & 0 \\
3 & 1 & 0 & 0 & 0 \\
4 & 3 & 1 & 0 & 0 \\
7 & 4 & 3 & 1 & 0 \\
11 & 7 & 4 & 3 & 1
\end{array}\right] \text { and } \mathscr{R}_{5}=\left[\begin{array}{ccccc}
1 & 3 & 4 & 7 & 11 \\
3 & 10 & 15 & 25 & 40 \\
4 & 15 & 26 & 43 & 69 \\
7 & 25 & 43 & 75 & 120 \\
11 & 40 & 69 & 120 & 196
\end{array}\right]
$$

From (1.5) and (1.6), we can see that $r_{1, j}=r_{j, 1}=L_{j}$.

Here, we note that the definition of the Lucas matrix and its inverse were given directly in [11]. However, the factorization of this matrix was not given by using the $(0,1,2)$ matrix whose entries are 0,1 and 2 .

In this paper, we fulfill this gap.

The set of all $n$-square matrices is denoted by $\mathscr{M}_{n}$. Let $B \in \mathscr{M}_{n}$. If the matrix $B$ can be written as $B=C C^{T}$ or $B=C^{T} C$, where $C$ is lower triangular matrix with nonnegative diagonal entries, then this factorization is called as Cholesky factorization. It is known that if $B$ is nonsingular, then this factorization is unique.

A matrix $A \in \mathscr{M}_{n}$ of the form

$$
A=\left[\begin{array}{cccc}
A_{11} & 0 & & \\
0 & A_{22} & & 0 \\
& & \ddots & \\
& 0 & & A_{k k}
\end{array}\right]
$$

in which $A_{i i} \in \mathscr{M}_{n_{i}}, i=1,2, \ldots, k$ and $\sum_{i=1}^{k} n_{i}=n$, is called block diagonal. This type of matrix is indicated as $A=A_{11} \oplus A_{22} \oplus \ldots \oplus A_{k k}$.

\section{Factorization of the Lucas matrix}

In [7], the authors gave the Cholesky factorization of the Fibonacci matrix. Motivated by this paper, we find the factorization of the Lucas matrix.

Let $I_{n}$ be the $n \times n$ identity matrix. We define the matrices $S_{n}, \overline{\mathscr{L}_{n}}$ and $G_{k}$ as

$$
S_{0}=\left[\begin{array}{lll}
1 & 0 & 0 \\
1 & 1 & 0 \\
1 & 0 & 1
\end{array}\right], S_{-1}=\left[\begin{array}{lll}
1 & 0 & 0 \\
0 & 1 & 0 \\
0 & 1 & 1
\end{array}\right],
$$

and $S_{k}=S_{0} \oplus I_{k}, k=1,2, \ldots, \overline{\mathscr{L}_{k}}=[1] \oplus \mathscr{L}_{n-1}, G_{1}=I_{n}, G_{2}=I_{n-3} \oplus S_{-1}$, and $G_{k}=I_{n-k} \oplus S_{k-3}$ for $k \geqslant 3$.

Now, we define factor matrix as

$$
W_{n}=\left[w_{i j}\right]=\left\{\begin{array}{lc}
1, & i=j \\
2, & i=j+1 \\
0, & \text { otherwise }
\end{array} .\right.
$$

By using the matrices $G_{k}$ and $W_{n}$, we have the following theorem. 
Theorem 2.1. The Lucas matrix $\mathscr{L}_{n}$ can be factored by the $G_{k}$ 's and $W_{n}$ as follows:

$$
\begin{aligned}
\mathscr{L}_{n} & =G_{1} G_{2} \cdots G_{n} W_{n} \\
& =W_{n} G_{1} G_{2} \cdots G_{n} .
\end{aligned}
$$

For example,

$$
\begin{aligned}
\mathscr{L}_{5}= & W_{5} G_{1} G_{2} G_{3} G_{4} G_{5} \\
= & {\left[\begin{array}{lllll}
1 & 0 & 0 & 0 & 0 \\
2 & 1 & 0 & 0 & 0 \\
0 & 2 & 1 & 0 & 0 \\
0 & 0 & 2 & 1 & 0 \\
0 & 0 & 0 & 2 & 1
\end{array}\right]\left[\begin{array}{lllll}
1 & 0 & 0 & 0 & 0 \\
0 & 1 & 0 & 0 & 0 \\
0 & 0 & 1 & 0 & 0 \\
0 & 0 & 0 & 1 & 0 \\
0 & 0 & 0 & 1 & 1
\end{array}\right]\left[\begin{array}{lllll}
1 & 0 & 0 & 0 & 0 \\
0 & 1 & 0 & 0 & 0 \\
0 & 0 & 1 & 0 & 0 \\
0 & 0 & 1 & 1 & 0 \\
0 & 0 & 1 & 0 & 1
\end{array}\right] } \\
& {\left[\begin{array}{lllll}
1 & 0 & 0 & 0 & 0 \\
0 & 1 & 0 & 0 & 0 \\
0 & 1 & 1 & 0 & 0 \\
0 & 1 & 0 & 1 & 0 \\
0 & 0 & 0 & 0 & 1
\end{array}\right]\left[\begin{array}{lllll}
1 & 0 & 0 & 0 & 0 \\
1 & 1 & 0 & 0 & 0 \\
1 & 0 & 1 & 0 & 0 \\
0 & 0 & 0 & 1 & 0 \\
0 & 0 & 0 & 0 & 1
\end{array}\right] } \\
= & {\left[\begin{array}{ccccc}
1 & 0 & 0 & 0 & 0 \\
3 & 1 & 0 & 0 & 0 \\
4 & 3 & 1 & 0 & 0 \\
7 & 4 & 3 & 1 & 0 \\
11 & 7 & 4 & 3 & 1
\end{array}\right] . }
\end{aligned}
$$

Now, we give other factorization of $\mathscr{L}_{n}$. Define $n \times n$ matrix $C_{n}=\left[c_{i j}\right]$ by

$$
c_{i j}=\left[\begin{array}{cccc}
L_{1} & 0 & \cdots & 0 \\
L_{2} & 1 & \cdots & 0 \\
\vdots & \vdots & \ddots & \vdots \\
L_{n} & 0 & \cdots & 1
\end{array}\right]
$$

Theorem 2.2. For $n \geqslant 2$, the Lucas matrix $\mathscr{L}_{n}$ can be factored by the $C_{n}$ 's as

$$
\mathscr{L}_{n}=C_{n}\left(I_{1} \oplus C_{n-1}\right)\left(I_{2} \oplus C_{n-2}\right) \cdots\left(I_{n-2} \oplus C_{2}\right) .
$$

In order to find factorization of the inverse Lucas matrix, we need inverse of the factor matrix. So, the following lemma explains inverse of the factor matrix $W_{n}$.

Lemma 2.3. Let $k$ be the non-negative integer and $W_{n}^{-1}=\left[w_{i j}^{\prime}\right]$ be the inverse of the matrix $W_{n}$. Then

$$
w_{i j}^{\prime}=\left\{\begin{array}{cc}
0, & i<j, \\
(-2)^{k} & i=j+k
\end{array}\right.
$$

holds.

For example,

$$
W_{5}^{-1}=\left[\begin{array}{ccccc}
1 & 0 & 0 & 0 & 0 \\
-2 & 1 & 0 & 0 & 0 \\
4 & -2 & 1 & 0 & 0 \\
-8 & 4 & -2 & 1 & 0 \\
16 & -8 & 4 & -2 & 1
\end{array}\right]
$$

Proof. Let $q_{i j}=\sum_{k=1}^{n} w_{i k} w_{k j}^{\prime}$. Obviously, $q_{i i}=1$ and $q_{i j}=0$ for $i<j$. For $i>j$,

$$
q_{i j}=2(-2)^{k}+1(-2)^{k+1}=0
$$

follows. This proves the lemma. 
The inverses of the matrices $S_{0}$ and $S_{1}$ are given in [7] as follows:

$$
S_{0}^{-1}=\left[\begin{array}{ccc}
1 & 0 & 0 \\
-1 & 1 & 0 \\
-1 & 0 & 1
\end{array}\right] \quad \text { and } S_{-1}^{-1}=\left[\begin{array}{ccc}
1 & 0 & 0 \\
0 & 1 & 0 \\
0 & -1 & 1
\end{array}\right] \text {. }
$$

We know that $S_{k}^{-1}=S_{0}^{-1} \oplus I_{k}$. Define $H_{k}=G_{k}^{-1}$. Then

$$
H_{1}=G_{1}^{-1}=I_{n}, H_{2}=G_{2}^{-1}=I_{n-3} \oplus S_{-1}^{-1} \text { and } H_{n}=S_{n-3}^{-1} .
$$

We also know that

$$
C_{n}^{-1}=\left[\begin{array}{cccc}
L_{1} & 0 & \cdots & 0 \\
-L_{2} & 1 & \cdots & 0 \\
\vdots & \vdots & \ddots & \vdots \\
-L_{n} & 0 & \cdots & 1
\end{array}\right] \text { and }\left(I_{k} \oplus C_{n-k}\right)^{-1}=I_{k} \oplus C_{n-k}^{-1}
$$

We know that inverse of the Lucas matrix is given directly by (see [11] p. 459, Theorem $2.2)$.

$$
\mathscr{L}_{n}^{-1}=\left[l_{i j}^{\prime}\right]=\left\{\begin{array}{cc}
1, & \text { if } i=j \\
-3, & \text { if } i=j+1 \\
5(-1)^{i-j} 2^{i-j-2}, & \text { if } i \geqslant j+2 \\
0, & \text { otherwise }
\end{array} .\right.
$$

Here, we find inverse of the Lucas matrix by using the matrices $G_{k}^{-1}$ and $W_{n}^{-1}$. Thus, the following theorem explains the factorization of the inverse Lucas matrix.

Theorem 2.4. The inverse of the Lucas matrix $\mathscr{L}_{n}^{-1}$ can be factored by the $G_{k}^{-1}$ 's and $W_{n}^{-1}$ as

$$
\begin{aligned}
\mathscr{L}_{n}^{-1} & =G_{n}^{-1} G_{n-1}^{-1} \cdots G_{2}^{-1} G_{1}^{-1} W_{n}^{-1} \\
& =H_{n} H_{n-1} \cdots H_{2} H_{1} W_{n}^{-1} \\
& =\left(I_{n-2} \oplus C_{2}\right)^{-1} \cdots\left(I_{1} \oplus C_{n-1}\right)^{-1} C_{n}^{-1} .
\end{aligned}
$$

For example, we have

$$
\begin{aligned}
\mathscr{L}_{5}^{-1}= & {\left[\begin{array}{ccccc}
1 & 0 & 0 & 0 & 0 \\
-1 & 1 & 0 & 0 & 0 \\
-1 & 0 & 1 & 0 & 0 \\
0 & 0 & 0 & 1 & 0 \\
0 & 0 & 0 & 0 & 1
\end{array}\right]\left[\begin{array}{ccccc}
1 & 0 & 0 & 0 & 0 \\
0 & 1 & 0 & 0 & 0 \\
0 & -1 & 1 & 0 & 0 \\
0 & -1 & 0 & 1 & 0 \\
0 & 0 & 0 & 0 & 1
\end{array}\right]\left[\begin{array}{ccccc}
1 & 0 & 0 & 0 & 0 \\
0 & 1 & 0 & 0 & 0 \\
0 & 0 & 1 & 0 & 0 \\
0 & 0 & -1 & 1 & 0 \\
0 & 0 & -1 & 0 & 1
\end{array}\right] } \\
& {\left[\begin{array}{ccccc}
1 & 0 & 0 & 0 & 0 \\
0 & 1 & 0 & 0 & 0 \\
0 & 0 & 1 & 0 & 0 \\
0 & 0 & 0 & 1 & 0 \\
0 & 0 & 0 & -1 & 1
\end{array}\right]\left[\begin{array}{ccccc}
1 & 0 & 0 & 0 & 0 \\
-2 & 1 & 0 & 0 & 0 \\
4 & -2 & 1 & 0 & 0 \\
-8 & 4 & -2 & 1 & 0 \\
16 & -8 & 4 & -2 & 1
\end{array}\right] } \\
= & {\left[\begin{array}{ccccc}
1 & 0 & 0 & 0 & 0 \\
-3 & 1 & 0 & 0 & 0 \\
5 & -3 & 1 & 0 & 0 \\
-10 & 5 & -3 & 1 & 0 \\
20 & -10 & 5 & -3 & 1
\end{array}\right] . }
\end{aligned}
$$

Now, we give the following lemma by using the definition of the symmetric Lucas matrix.

Lemma 2.5. Each entries of the matrix $\mathscr{R}_{n}=\left[r_{i, j}\right]$ can be expressed by

$$
r_{i, j}= \begin{cases}L_{i+j+1}-L_{j-i+1+\xi(i)}, & \text { if } j \geqslant i \\ L_{i+j+1}-L_{i-j+1+\xi(j)}, & \text { if } j<i\end{cases}
$$


where $\xi(k)=k-2\left\lfloor\frac{k}{2}\right\rfloor$.

Proof. One can use the induction method together with the definition of the Lucas matrix.

Before giving the Cholesky factorization of the matrix $\mathscr{R}_{n}$, we need to give the following main theorem.

Theorem 2.6. If $1 \leqslant i \leqslant j$, then

$$
5 \sum_{t=1}^{i-2}(-2)^{i-2-t} r_{t, j}-3 r_{i-1, j}+r_{i, j}=L_{j-i+1}
$$

holds. Otherwise, we have

$$
5 \sum_{t=1}^{i-2}(-2)^{i-2-t} r_{t, j}-3 r_{i-1, j}+r_{i, j}=0
$$

Proof. Assume that $1 \leqslant i \leqslant j$. We use the induction method in order to prove the theorem. From the definition of (1.6), we observe that $r_{i, j+2}=r_{i, j+1}+r_{i, j}$. This finishes the induction on $j$.

Now, we focus on the induction on $i$. Since $5 \sum_{t=1}^{i-2}(-2)^{i-2-t} r_{t, j}=0$, the equation (2.3) is true for $i=1,2$. For $i=3$ in the equation (2.3),

$$
\begin{aligned}
5 r_{1, j}-3 r_{2, j}+r_{3, j} & =5\left(L_{j+2}-L_{j+1}\right)-3\left(L_{j+3}-L_{j-1}\right)+L_{j+4}-L_{j-1} \\
& =L_{j+4}-3 L_{j+3}+5 L_{j}+2 L_{j-1} \\
& =L_{j-2}
\end{aligned}
$$

holds as claimed. Now, suppose that it is true for all integer $i \geqslant 4$. By using the equation (2.3) and induction hypothesis, we have

$$
\begin{aligned}
5 \sum_{t=1}^{i-1}(-2)^{i-1-t} r_{t, j}-3 r_{i, j}+r_{i+1, j} & =5\left(r_{i-1, j}-2 \sum_{t=1}^{i-2}(-2)^{i-2-t} r_{t, j}\right)-3 r_{i, j}+r_{i+1, j} \\
& =5 r_{i-1, j}-2\left(L_{j-i+1}+3 r_{i-1, j}-r_{i, j}\right)-3 r_{i, j}+r_{i+1, j} \\
& =-r_{i-1, j}+r_{i+1, j}-r_{i, j}-2 L_{j-i+1} .
\end{aligned}
$$

From Lemma 2.5, we know that $r_{i, j}=L_{i+j+1}-L_{j-i+1+\xi(i)}$. Therefore we have

$$
\begin{aligned}
5 \sum_{t=1}^{i-1}(-2)^{i-1-t} r_{t, j}-3 r_{i, j}+r_{i+1, j}= & -r_{i-1, j}+r_{i+1, j}-r_{i, j}-2 L_{j-i+1} \\
= & -L_{i+j}-L_{i+j+1}+L_{i+j+2}-2 L_{j-i+1} \\
& +L_{j-i+2+\xi(i-1)}+L_{j-i+1+\xi(i)}-L_{j-i+\xi(i+1)} \\
= & L_{j-i+1+\xi(i+1)}+L_{j-i+1+\xi(i)}-2 L_{j-i+1} \\
= & L_{j-i+3}-2 L_{j-i+1} \\
= & L_{j-i} .
\end{aligned}
$$

The other case $j<i$ can be proven similarly. Therefore, we omit the details. So, the proof is completed.

Theorem 2.7. For $n \geqslant 1$ positive integer, $H_{n} H_{n-1} \ldots H_{1} W_{n}^{-1} \mathscr{R}_{n}=\mathscr{L}_{n}^{T}$ and the Cholesky factorization is given by $\mathscr{R}_{n}=\mathscr{L}_{n} \mathscr{L}_{n}^{T}$.

Proof. Together with the facts $H_{n} H_{n-1} \ldots H_{1} W_{n}^{-1}=\mathscr{L}_{n}^{-1}$ and $\mathscr{L}_{n}^{-1} \mathscr{R}_{n}=\mathscr{L}_{n}^{T}$, we have $\mathscr{R}_{n}=\mathscr{L}_{n} \mathscr{L}_{n}^{T}$. This gives the Cholesky factorization of the matrix $\mathscr{R}_{n}$. 
In particular, with the help of Theorem 2.7, we can give the Cholesky factorization of the inverse symmetric Lucas matrix as $\mathscr{R}_{n}^{-1}=\left(\mathscr{L}_{n}^{T}\right)^{-1} \mathscr{L}_{n}^{-1}=\left(\mathscr{L}_{n}^{-1}\right)^{T} \mathscr{L}_{n}^{-1}$. For example,

$$
\mathscr{R}_{7}^{-1}=\left[\begin{array}{ccccccc}
8535 & -4268 & 2135 & -1070 & 540 & -280 & 80 \\
-4268 & 2135 & -1068 & 535 & -270 & 140 & -40 \\
2135 & -1068 & 535 & -268 & 135 & -70 & 20 \\
-1070 & 535 & -268 & 135 & -68 & 35 & -10 \\
540 & -270 & 135 & -68 & 35 & -18 & 5 \\
-280 & 140 & -70 & 35 & -18 & 10 & -3 \\
80 & -40 & 20 & -10 & 5 & -3 & 1
\end{array}\right] .
$$

\section{Eigenvalues of $\mathscr{R}_{n}$}

In this section, we consider the eigenvalues of the symmetric Lucas matrix $\mathscr{R}_{n}$.

Let $\Omega$ be an $n \times n$ matrix. The authors, in [4], stated that if $\Omega$ is an $n \times n$ Hermitian matrix then it is positive definite if and only if $\operatorname{det} \Omega>0$. For $n \geqslant 2$, we observe that $\mathscr{R}_{n}$ is Hermitian and by Theorem 2.7 , we have $\operatorname{det} \mathscr{R}_{n}=\operatorname{det}\left(\mathscr{L}_{n} \mathscr{L}_{n}^{T}\right)=1$. Hence, $\mathscr{R}_{n}$ is a positive definite matrix and therefore the eigenvalues of $\mathscr{R}_{n}$ are all positive.

Let $\mathscr{D}=\left\{x=\left(x_{1}, x_{2}, \ldots, x_{n}\right) \in \mathbb{R}^{n} ; x_{1} \geqslant x_{2} \geqslant \ldots \geqslant x_{n}\right\}$. For $x, y \in \mathscr{D}$,

$$
x \prec y, \quad \text { if } \quad\left\{\begin{array}{l}
\sum_{j=1}^{i} x_{j} \leqslant \sum_{j=1}^{i} y_{j}, \quad i=1,2, \ldots, n-1, \\
\sum_{j=1}^{n} x_{j}=\sum_{j=1}^{n} y_{j}
\end{array}\right.
$$

When $x \prec y, x$ is said to be majorized by $y$ or $y$ is said to majorize $x$. On the other hand, the condition for majorization can be rewritten as follows:

$$
x \prec y, \quad \text { if } \quad\left\{\begin{array}{l}
\sum_{j=0}^{i} x_{n-j} \geqslant \sum_{j=0}^{i} y_{n-j}, \quad i=0,1, \ldots, n-2, \\
\sum_{j=0}^{n-1} x_{n-j}=\sum_{j=0}^{n-1} y_{n-j} .
\end{array} .\right.
$$

Note that, there is an interesting simple fact as follows:

$$
(\bar{x}, \bar{x}, \ldots, \bar{x}) \prec\left(x_{1}, x_{2}, \ldots, x_{n}\right),
$$

where $\bar{x}=\frac{\sum_{i=1}^{n} x_{i}}{n}$. For more information about majorizations, we refer to the book [9].

For $i, j=1,2, \ldots, n$, an $n \times n$ matrix $\Phi_{n}=\left[\phi_{i j}\right]$ is a doubly stochastic matrix if $\phi_{i j} \geqslant 0$, $\sum_{i=1}^{n} \phi_{i j}=1$ and $\sum_{j=1}^{n} \phi_{i j}=1$. Hardy, Littlewood and Pólya [3], stated that a necessary and sufficient condition that $x \prec y$ is that there exists a doubly stochastic matrix $\Phi_{n}$ such that $x=y \Phi_{n}$.

Note that $\operatorname{det} \mathscr{L}_{n}=1$ and $\operatorname{det} \mathscr{R}_{n}=1$. Let $\lambda_{1}, \lambda_{2}, \ldots, \lambda_{n}$ be the eigenvalues of $\mathscr{R}_{n}$. We know that $\mathscr{R}_{n}=\mathscr{L}_{n} \mathscr{L}_{n}^{T}$ and $\sum_{i=1}^{k} L_{i}^{2}=\left(L_{k+1} L_{k}-2\right)$, all of the eigenvalues of $\mathscr{R}_{n}$ are positive and

$$
\left(L_{n+1} L_{n}-2, L_{n} L_{n-1}-2, \ldots, L_{2} L_{1}-2\right) \prec\left(\lambda_{1}, \lambda_{2}, \ldots, \lambda_{n}\right) .
$$

So, we have the following corollaries.

Corollary 3.1. Let $\lambda_{1}, \lambda_{2}, \ldots, \lambda_{n}$ be the eigenvalues of $\mathscr{R}_{n}$. Then

$$
\lambda_{1}+\lambda_{2}+\cdots+\lambda_{n}=L_{2 n+2}-2 n-3-\xi(n) .
$$

Proof. By virtue of $\left(L_{n+1} L_{n}-2, L_{n} L_{n-1}-2, \ldots, L_{2} L_{1}-2\right) \prec\left(\lambda_{1}, \lambda_{2}, \ldots, \lambda_{n}\right)$, we get

$$
\lambda_{1}+\lambda_{2}+\cdots+\lambda_{n}=\left\{\begin{array}{ll}
\left(L_{n+1}\right)^{2}-2 n-1, & \text { if } n \text { is even } \\
\left(L_{n+1}\right)^{2}-2 n-6, & \text { if } n \text { is odd }
\end{array}=L_{2 n+2}-2 n-3-\xi(n) .\right.
$$


Corollary 3.2. Let $\lambda_{1}, \lambda_{2}, \ldots, \lambda_{n}$ be the eigenvalues of $\mathscr{R}_{n}$. Then

$$
n \lambda_{n} \leqslant L_{2 n+2}-2 n-3-\xi(n) \leqslant n \lambda_{1} .
$$

Proof. Let $s_{n}=\lambda_{1}+\lambda_{2}+\cdots+\lambda_{n}$. Since

$$
\left(\frac{s_{n}}{n}, \frac{s_{n}}{n}, \ldots, \frac{s_{n}}{n}\right) \prec\left(\lambda_{1}, \lambda_{2}, \ldots, \lambda_{n}\right),
$$

we get $\lambda_{n} \leqslant \frac{s_{n}}{n} \leqslant \lambda_{1}$. Hence, the proof is completed.

From (2.4), we get

$$
\left(10+5^{2}\left(\frac{2^{2 n-4}-1}{3}\right), 10+5^{2}\left(\frac{2^{2 n-6}-1}{3}\right), \ldots, 35,10,1\right) \prec\left(\frac{1}{\lambda_{n}}, \frac{1}{\lambda_{n-1}}, \ldots, \frac{1}{\lambda_{2}}, \frac{1}{\lambda_{1}}\right) .
$$

Hence, there exists a doubly stochastic matrix $\Phi_{n}=\left[\varphi_{i j}\right]$ such that

$$
\begin{aligned}
\left(10+5^{2}\left(\frac{2^{2 n-4}-1}{3}\right), 10+5^{2}\left(\frac{2^{2 n-6}-1}{3}\right), \ldots, 35,10,1\right) & \\
= & \left(\frac{1}{\lambda_{n}}, \frac{1}{\lambda_{n-1}}, \ldots, \frac{1}{\lambda_{2}}, \frac{1}{\lambda_{1}}\right)\left[\begin{array}{cccc}
\varphi_{11} & \varphi_{12} & \ldots & \varphi_{1 n} \\
\varphi_{21} & \varphi_{22} & \ldots & \varphi_{2 n} \\
\vdots & \vdots & \ldots & \vdots \\
\varphi_{n 1} & \varphi_{n 2} & \ldots & \varphi_{n n}
\end{array}\right] .
\end{aligned}
$$

Namely, we have $\frac{1}{\lambda_{n}} \varphi_{1 n}+\frac{1}{\lambda_{n-1}} \varphi_{2 n}+\cdots+\frac{1}{\lambda_{1}} \varphi_{n n}=1$ and $\varphi_{1 n}+\varphi_{2 n}+\cdots+\varphi_{n n}=1$.

Lemma 3.3. For each $i=1,2, \ldots, n$, we have $\varphi_{n-(i-1), n} \leqslant \frac{\lambda_{i}}{n-1}$.

Proof. Suppose that $\varphi_{n-(i-1), n}>\frac{\lambda_{i}}{n-1}$. Then

$$
\varphi_{1 n}+\varphi_{2 n}+\cdots+\varphi_{n n}>\frac{\lambda_{1}}{n-1}+\frac{\lambda_{2}}{n-1}+\cdots \frac{\lambda_{n}}{n-1}=\frac{1}{n-1}\left(\lambda_{1}+\lambda_{2}+\cdots+\lambda_{n}\right) .
$$

Since $\sum_{i=1}^{n} \varphi_{i n}=1$ and $\sum_{i=1}^{n} \lambda_{i} \geqslant n$, this yields a contradiction, so $\varphi_{n-(i-1), n} \leqslant \frac{\lambda_{i}}{n-1}$.

Let

$$
\tau=\frac{1}{n}\left(1+10+35+\ldots+10+5^{2}\left(\frac{2^{2 n-4}-1}{3}\right)\right)=\frac{5^{2} 4^{n-1}+15(n-2)-1}{9 n}=\frac{1}{n} \sum_{i=1}^{n} \frac{1}{\lambda_{i}} .
$$

Then, we have

$$
(\tau, \tau, \ldots, \tau) \prec\left(\frac{1}{\lambda_{n}}, \frac{1}{\lambda_{n-1}}, \ldots, \frac{1}{\lambda_{1}}\right) .
$$

The next theorem explains the majorization of the eigenvalues of $\mathscr{R}_{n}$.

Theorem 3.4. For $\left(\lambda_{1}, \lambda_{2}, \ldots, \lambda_{n}\right) \in \mathscr{D}$, we have

$$
\left(\frac{1}{n-1}\left(s_{n}-\frac{1}{\tau}\right), \ldots, \frac{1}{n-1}\left(s_{n}-\frac{1}{\tau}\right), \frac{1}{\tau}\right) \prec\left(\lambda_{1}, \lambda_{2}, \ldots, \lambda_{n}\right) .
$$

Proof. Let $\Psi_{n}$ be an $n \times n$ matrix as follows:

where

$$
\Psi_{n}=\left[\begin{array}{ccccc}
\frac{1-\psi_{1 n}}{n-1} & \frac{1-\psi_{1 n}}{n-1} & \ldots & \frac{1-\psi_{1 n}}{n-1} & \psi_{1 n} \\
\frac{1-\psi_{2 n}}{n-1} & \frac{1-\psi_{2 n}}{n-1} & \ldots & \frac{1-\psi_{2 n}}{n-1} & \psi_{2 n} \\
\vdots & \vdots & \vdots & & \vdots \\
\frac{1-\psi_{n n}}{n-1} & \frac{1-\psi_{n n}}{n-1} & \cdots & \frac{1-\psi_{n n}}{n-1} & \psi_{n n}
\end{array}\right]
$$




$$
\psi_{i j}=\frac{1-\psi_{i n}}{n-1}, \quad i=1,2, \ldots, n, \quad j=1,2, \ldots, n-1
$$

and

$$
\psi_{\text {in }}=\frac{1}{n \tau \lambda_{i}}, \quad i=1,2, \ldots, n .
$$

Then, for $i=1,2, \ldots, n$, we observe that $\psi_{i n} \geqslant 0$ and

$$
\begin{gathered}
\psi_{1 n}+\psi_{2 n}+\cdots+\psi_{n n}=\frac{1}{n \tau \lambda_{1}}+\frac{1}{n \tau \lambda_{2}}+\cdots+\frac{1}{n \tau \lambda_{n}}=1, \\
(n-1) \frac{1-\psi_{i n}}{n-1}+\psi_{i n}=1
\end{gathered}
$$

and

$$
\frac{1-\psi_{1 n}}{n-1}+\frac{1-\psi_{2 n}}{n-1}+\cdots+\frac{1-\psi_{n n}}{n-1}=\frac{1}{n-1}\left(n-\left(\psi_{1 n}+\psi_{2 n}+\cdots+\psi_{n n}\right)\right)=1 .
$$

Therefore, $\Psi_{n}$ is a doubly stochastic matrix. Moreover, we have

$$
\lambda_{1} \psi_{1 n}+\lambda_{2} \psi_{2 n}+\cdots+\lambda_{n} \psi_{n n}=\frac{1}{\tau}
$$

and

$$
\begin{aligned}
\lambda_{1} \frac{1-\psi_{1 n}}{n-1}+\lambda_{2} \frac{1-\psi_{2 n}}{n-1}+\cdots+\lambda_{n} \frac{1-\psi_{n n}}{n-1} & =\frac{1}{n-1}\left(s_{n}-\left(\lambda_{1} \psi_{1 n}+\lambda_{2} \psi_{2 n}+\cdots+\lambda_{n} \psi_{n n}\right)\right) \\
& =\frac{1}{n-1}\left(s_{n}-\frac{1}{\tau}\right) .
\end{aligned}
$$

Hence, we get

$$
\left(\frac{1}{n-1}\left(s_{n}-\frac{1}{\tau}\right), \ldots, \frac{1}{n-1}\left(s_{n}-\frac{1}{\tau}\right), \frac{1}{\tau}\right)=\left(\lambda_{1}, \lambda_{2}, \ldots, \lambda_{n}\right) \Psi_{n} .
$$

As a result, we have

$$
\left(\frac{1}{n-1}\left(s_{n}-\frac{1}{\tau}\right), \ldots, \frac{1}{n-1}\left(s_{n}-\frac{1}{\tau}\right), \frac{1}{\tau}\right) \prec\left(\lambda_{1}, \lambda_{2}, \ldots, \lambda_{n}\right) .
$$

By virtue of equation (2.4), we have the following lemma which explains the lower bounds of the eigenvalues of $\mathscr{R}_{n}$.

Lemma 3.5. For $k=2,3, \ldots, n$, we have

$$
\frac{1}{\gamma_{k}} \leqslant \lambda_{k}
$$

where $\gamma_{k}=\frac{5^{2} 4^{k-1}+15(k-2)-1}{9}$ is sum of the diagonal elements of $\mathscr{R}^{-1}$.

Proof. By virtue of (2.4), we have

$$
\frac{1}{\lambda_{1}}+\frac{1}{\lambda_{2}}+\cdots+\frac{1}{\lambda_{k}} \leqslant 1+10+35+\ldots+10+5^{2}\left(\frac{2^{2 k-4}-1}{3}\right)=\gamma_{k} .
$$


Hence,

$$
\frac{1}{\lambda_{k}} \leqslant \gamma_{k}-(\underbrace{\frac{1}{\lambda_{1}}+\frac{1}{\lambda_{2}}+\cdots+\frac{1}{\lambda_{k-1}}}_{\geqslant 0}) \leqslant \gamma_{k} .
$$

Therefore, we have $\frac{1}{\gamma_{k}} \leqslant \lambda_{k}$.

In the following theorem, we give some upper and lower bounds for the eigenvalues of $\mathscr{R}_{n}$.

Theorem 3.6. For $k=1,2, \ldots, n-2$, we have

$$
\frac{1}{\gamma_{n-k}} \leqslant \lambda_{n-k} \leqslant \frac{1}{n-1}\left(\frac{n-k-1}{\tau}+k s_{n}\right)-\sum_{i=0}^{k-1} \frac{1}{\gamma_{n-i}} .
$$

In particular,

$$
\frac{1}{n-1}\left(s_{n}-\frac{1}{\tau}\right) \leqslant \lambda_{1} \leqslant \prod_{i=2}^{n} \gamma_{i}
$$

and

$$
\frac{1}{\gamma_{n}} \leqslant \lambda_{n} \leqslant \frac{1}{\tau}
$$

Proof. By virtue of Theorem 3.4, we observe that $\frac{1}{n-1}\left(s_{n}-\frac{1}{\tau}\right) \leqslant \lambda_{1}$ and $\lambda_{n} \leqslant \frac{1}{\tau}$. From Lemma 3.5 , we have $\frac{1}{\gamma_{n}} \leqslant \lambda_{n}$. Since $\operatorname{det} \mathscr{R}_{n}=\operatorname{det}\left(\mathscr{L}_{n} \mathscr{L}_{n}^{T}\right)=1=\lambda_{1} \lambda_{2} \ldots \lambda_{n}$, by Lemma 3.5 , we have

$$
\lambda_{1} \prod_{i=2}^{n} \frac{1}{\gamma_{i}} \leqslant \lambda_{1} \lambda_{2} \lambda_{3} \ldots \lambda_{n}=1 .
$$

Thus, we obtain $\lambda_{1} \leqslant \prod_{i=2}^{n} \gamma_{i}$. From Theorem 3.4, we have

$$
\begin{aligned}
\lambda_{n}+\lambda_{n-1}+\cdots+\lambda_{n-k} & \leqslant \frac{1}{\tau}+\frac{k}{n-1}\left(s_{n}-\frac{1}{\tau}\right) \\
& =\frac{1}{n-1}\left(\frac{n-k-1}{\tau}+k s_{n}\right) .
\end{aligned}
$$

Thus, by using Lemma 3.5, we obtain

$$
\begin{aligned}
\lambda_{n-k} & \leqslant \frac{1}{n-1}\left(\frac{n-k-1}{\tau}+k s_{n}\right)-\left(\lambda_{n}+\lambda_{n-1}+\cdots+\lambda_{n-k+1}\right) \\
& \leqslant \frac{1}{n-1}\left(\frac{n-k-1}{\tau}+k s_{n}\right)-\sum_{i=0}^{k-1} \frac{1}{\gamma_{n-i}} .
\end{aligned}
$$

Therefore, we obtain

$$
\frac{1}{\gamma_{n-k}} \leqslant \lambda_{n-k} \leqslant \frac{1}{n-1}\left(\frac{n-k-1}{\tau}+k s_{n}\right)-\sum_{i=0}^{k-1} \frac{1}{\gamma_{n-i}} .
$$

Acknowledgment. The authors thank to the anonymous reviewers for their insightful comments and suggestions. 


\section{References}

[1] C.M. Fonseca and E. Kılıç, An observation on the determinant of a Sylvester-Kac type matrix, An. Univ. "Ovidius" Constanta Ser. Mat. 28 (1), 111-115, 2020.

[2] C.M. Fonseca and Kılıç, A new type of Sylvester-Kac matrix and its spectrum, https://doi.org/10.1080/03081087.2019.1620673.

[3] G.H. Hardy, J.E. Littlewood and G. Pólya, Some simple inequalities satisfied by convex functions, Messenger Math. 58, 145-152, 1929.

[4] C.R. Johnson and R.A. Horn, Matrix analysis, Cambridge University Press Cambridge, 1985.

[5] E. Kilic and D. Tasci, The linear algebra of the Pell matrix, Bol. Soc. Mat. Mex. 11 (3), 163-174, 2005.

[6] T. Koshy, Fibonacci and Lucas Numbers with Applications, John Wiley \& Sons, 2001.

[7] G.-Y. Lee, J.-S. Kim and S.-G. Lee, Factorizations and eigenvalues of Fibonacci and symmetric Fibonacci matrices, Fibonacci Quart. 40 (3), 203-211, 2002.

[8] G.-Y. Lee and J.-S. Kim, The linear algebra of the $k$-Fibonacci matrix, Linear Algebra Appl. 373, 75-87, 2003.

[9] A.W. Marshall, I. Olkin and B.C. Arnold, Inequalities: theory of majorization and its applications 143, Springer, 1979.

[10] P. Stanica, Cholesky factorizations of matrices associated with $r$-order recurrent sequences, Integers, 5 (2), A16, 2005.

[11] Z. Zhang and Y. Zhang, The Lucas matrix and some combinatorial identities, Indian J. Pure Appl. Math. 38 (5), 457-465, 2007. 